\title{
RE-EVALUATION OF ASYNCHRONOUS ASEXUAL DEVELOPMENT OF CYSTOISOSPORA CANIS IN INTESTINES OF DOGS
}

\author{
J. P. Dubey ${ }^{1}$ and D. S. Lindsay ${ }^{2}$ \\ ${ }^{1}$ U.S. Department of Agriculture, Agricultural Research Service, Beltsville Agricultural Research Center, Animal Parasitic Diseases Laboratory, Beltsville, \\ Maryland 20705-2350. \\ 2 Department of Biomedical Science and Pathology, Virginia-Maryland Regional College of Veterinary Medicine, Virginia Tech, 1410 Prices Fork Road, \\ Blacksburg, Virginia 24061 . \\ Correspondence should be sent to J. P. Dubey at: jitender.dubey@ars.usda.gov
}

\begin{tabular}{|c|c|}
\hline KEY WORDS & ABSTRACT \\
\hline
\end{tabular}

Cystoisospora canis (syn. Isospora canis) is a coccidian that can cause clinical disease in dogs (Mitchell et al., 2007). Lepp and Todd (1974) described the endogenous development of a cloned culture of $C$. canis in coccidia-free dogs inoculated orally with 150,000 sporulated oocysts. One dog was examined at necropsy each day from 1 to 12 days postinoculation (PI). They reported 3 generations of schizonts. The first asexual stages (trophozoites) were seen days 1-3 PI and the first nuclear division was observed on day 4 PI. First-generation schizonts matured 5-7 days PI; they were 16-38 $\times 11-23 \mu \mathrm{m}$ and contained 4-24 merozoites measuring $8-11 \times 3-5 \mu \mathrm{m}$. The second-generation schizonts occurred 6-7 days PI, were $12-18 \times 8-13 \mu \mathrm{m}$, and contained 3-12 merozoites measuring $11-13 \times 3-5 \mu \mathrm{m}$. The third-generation schizonts occurred 6-8 days PI, were 13-38 $\times$ 8-24 $\mu \mathrm{m}$, and contained 672 merozoites measuring $8-13 \times 1.5-3.0 \mu \mathrm{m}$. The second and third generations were seen in the same parasitophorous vacuole. Gamonts were seen 7-10 days PI. No mention was made of schizonts occurring at 9-12 days PI.

In the present paper, we report asynchronous asexual development and multiple multinucleate schizonts/groups occurring in the same host cell in the small intestines of 2 dogs necropsied 10 days after feeding 100,000 C. canis oocysts.

The 2 dogs (AJV and AIZ2) studied here were used by Mitchell et al. (2007). The dogs were female beagles and obtained commercially (Covance, Cumberland, Virginia) at 6 wk of age. Feces of these dogs were examined by sugar flotation for 3 days before inoculation with sporulated $C$. canis oocysts. Coccidian oocysts were never detected in feces of these dogs before inoculation (Mitchell et al., 2007). Both dogs were each inoculated orally with 100,000 oocysts and euthanized 10 days later. The 

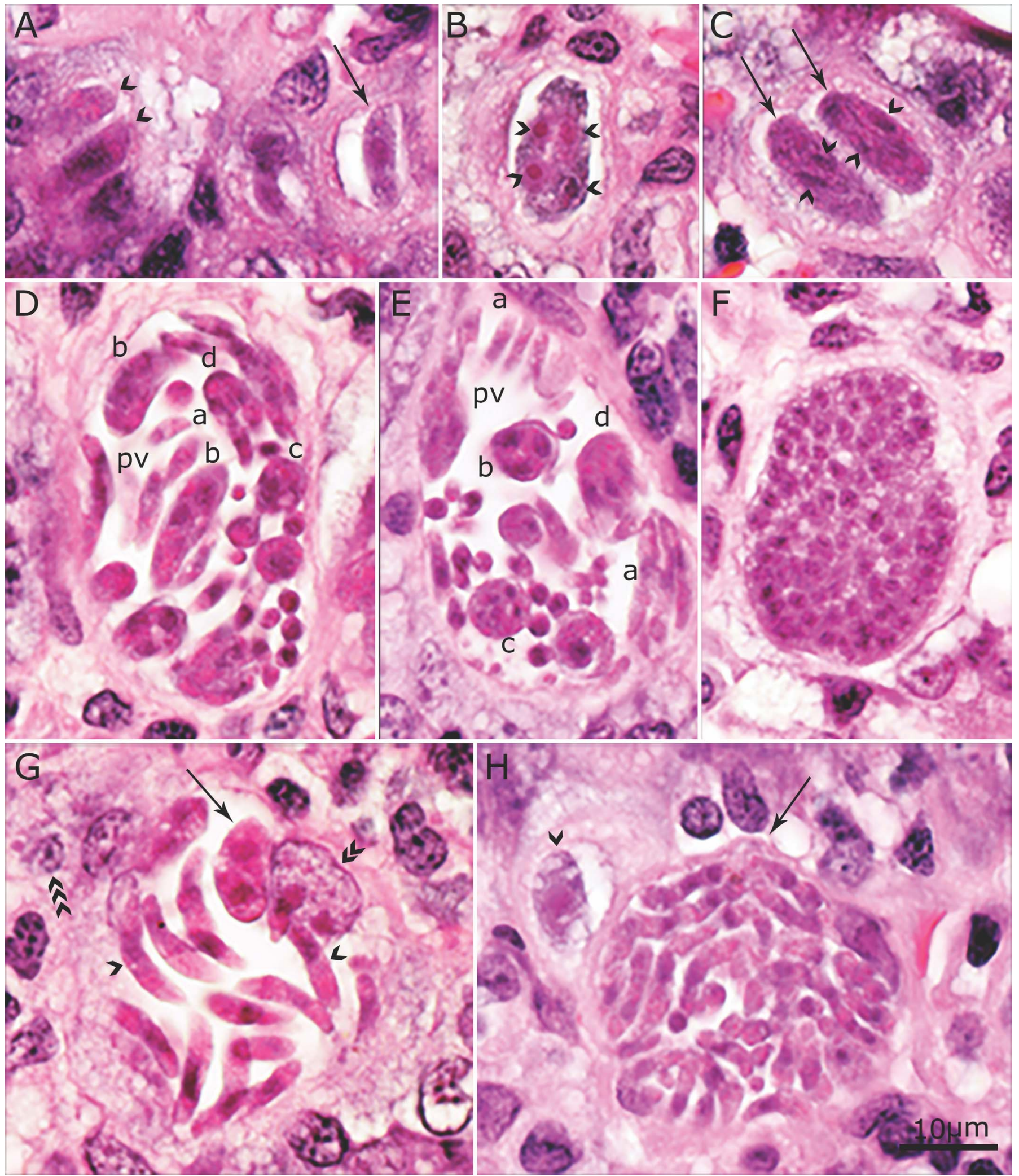

Figure 1. Asexual stages of Cystoisospora canis in the lamina propria of small intestine of dogs 10 days after oral inoculation with 100,000 oocysts Hematoxylin and eosin stain. Scale bar applies to all images in figure. (A) Single (arrow) and 2 merozoites (arrowheads) in a parasitophorous vacuoles (pv). (B) Parasite with 4 nuclei (arrowheads). (C) Developing merozoites in 2 meronts (arrows) within 1 pv. (D) Developing merozoites in a large pv. Note different shapes and sizes of merozoites including single (a), 2 crescentic meronts (b), pear-shaped meront (c), and merozoites budding from a meront (d). (E) Different-sized merozoites/ meronts in a pv. Note slender merozoites (a), meronts with 3 nuclei (b), meront with 5 or more nuclei (c), and merozoites budding off a meront (d). (F) A microgamont/schizont-like structure with 70 or more nuclei. (G) A group of merozoites (arrowheads) in a pv. Note 1 organism with 2 nuclei (arrow). (H) Meront with many merozoites (arrow). Arrowhead points to an undifferentiated uninucleated organism. Color version available online. 
strain of C. canis used was derived from feces of 2 pit bull puppies and oocysts from the experimentally infected dogs were used to redescribe morphology of $C$. canis oocysts (Mitchell et al., 2007). This strain of oocysts is no longer available because of loss of viability after $>1 \mathrm{yr}$ of storage at $4 \mathrm{C}$.

The dogs excreted C. canis oocysts with a prepatent period of 9 days. Loops of duodenum, jejunum, and ileum were fixed in formalin and processed for histological sections (Mitchell et al., 2007). For the present study, sections were recut from archived paraffin blocks and were examined after staining with hematoxylin and eosin. In total, 51 segments $(2-6 \mathrm{~cm}$ long) were examined at $\times 1,000$ magnification on an Olympus AX 70 microscope (Olympus America Inc., Melville, New York) and stages photographed using a DP73 camera.

Asexual and sexual stages were seen in the lamina propria throughout the small intestine. The parasitized host cell (probably enterocyte) nucleus was indented but rarely hypertrophied. Merozoites of different sizes were present, often in the same vacuole (Fig. 1). They were arranged singly, in pairs (Fig. 1A), and many within a vacuole (Fig. 1D, E). The maximum number of nuclei within developing merozoites in a group was 8 , but it could not be discerned if they were individual nuclei or parts of merozoites. The largest meront/group was $53 \times 35 \mu \mathrm{m}$. Rarely, there were groups with many nuclei (Fig. 1F). At least 80 nuclei are present in Figure $1 \mathrm{~F}$ but it is not clear if this is a schizont or microgamont. Individual merozoites were $12-15 \mu \mathrm{m}$ long and 3-6 $\mu \mathrm{m}$ wide. The parasite in Figure $1 \mathrm{~B}$ is $15.5 \times 8.5 \mu \mathrm{m}$, contains 4 nuclei, and appears to split into 2 merozoites, each with 2 nuclei. The merozoites varied in intensity of staining. Figure $1 \mathrm{G}$ contains merozoites that are more eosinophilic than those in Figure 1C. In 1 meront the merozoites were only $5 \mu \mathrm{m}$ long.

Findings of asexual stages 1 day after dogs had started excreting oocysts indicated continued asexual multiplication beyond the prepatent period. The stages found resemble the 3 generations reported by Lepp and Todd (1974). The mode of division of the asexual generations remains unclear. The study of Lepp and Todd (1974) is difficult to compare with our findings and those of others because limited quantitative data were presented. They did not provide the numbers of stages that they examined to determine a mean and a range. Lepp and Todd (1974) schematically depicted as many as 19 separate nuclei in a first-generation immature schizont and indicate that from 4 to 24 merozoites were in mature first-generation schizonts. They did not provide line drawings or micrographs of immature multinucleate second-generation schizonts. They reported that 3-12 merozoites were present in mature second-generation schizonts (Lepp and Todd, 1974). The numbers of merozoites in mature third-generation schizonts ranged from 6 to 72. Specimens (oocysts, histological sections) from the study of Lepp and Todd (1974) are not available now for re-evaluation (J. P. Dubey, pers. comm.).

Hilali et al. (1979) described the ultrastructure of schizonts and merozoites of $C$. canis in the intestines of dogs examined on day 5 PI. These would the first generation according to Lepp and Todd (1974) because second-generation schizonts were not present until day 6 PI. Hilali et al. (1979) found that C. canis divided by endodyogeny and they did not observe multinucleated stages.

Until the discovery of the full life cycle of Toxoplasma gondii as a coccidian parasite of cats, little was known of the coccidian parasites of dogs and cats (Dubey and Frenkel, 1972). The canid and felid coccidia were placed in the genus Isospora with oocysts containing 2 sporocysts, each with 4 sporozoites. Isospora species were thought to follow the life cycle of Eimeria species. In the Eimeria life cycle, the parasite undergoes a series of asexual multiplication cycles called generations (Levine, 1973). The sporozoite nucleus divides into 6 or more nuclei by schizogony and merozoites are formed synchronously, either internally or at the periphery. The merozoites from each successive generation are often morphologically different. Merozoites from the terminal generation form gamonts and the cycle is completed when oocysts are excreted in feces. Unlike Eimeria, T. gondii multiplied profusely asexually in cat intestinal enterocytes, and asexual multiplication continues long after oocysts were first excreted (Dubey and Frenkel, 1972). The parasite multiplies by endodyogeny, by splitting, and by schizogony. Additionally, parasites multiply asynchronously, and mature merozoites are seen along with immature stages. Dubey and Frenkel (1972) proposed the term "type" to replace generation.

With respect to other feline coccidia, Shah (1971), who studied the life cycle of $C$. felis, called the groups with more than 1 generation of schizonts "cysts." However, cyst is not an accurate term because it is best used for an orally infective stage of coccidia; monozoic tissue cysts of $C$. felis and other Cystoisospora spp. from cats and dogs can occur in paratenic hosts in extraintestinal organs (Lindsay et al., 2014). Subsequently, other feline and canine coccidia were found to divide by endodyogeny, with host cells containing products of more than 1 divisional cycle (Dubey, 1978, 1979, 2014; Dubey et al., 1978; Ferguson et al., 1980). The swine coccidian, C. suis, was found to divide first by endodyogeny repeatedly and then by schizogony, like feline and canine Cystoisospora spp., and the asexual development was described as types, not generations (Lindsay et al., 1980). Thus, it is more appropriate to use the term type for asexual multiplication for Cystoisospora spp. than generation.

We thank Camila Cezar for assistance.

\section{LITERATURE CITED}

Dubey, J. P. 1978. Life cycle of Isospora ohioensis in dogs. Parasitology 77: 1-11.

Dubey, J. P. 1979. Life cycle of Isospora rivolta (Grassi, 1879) in cats and mice. Journal of Protozoology 26: 433-443.

Dubey, J. P. 2014. Life cycle of Cystoisospora felis (Coccidia: Apicomplexa) in cats and mice. Journal of Eukaryotic Microbiology 61: 637-643.

Dubey, J. P., And J. K. Frenkel. 1972. Cyst-induced toxoplasmosis in cats. Journal of Protozoology 19: 155-177.

Dubey, J. P., S. E. Weisbrode, And W. A. Rogers. 1978. Canine coccidiosis attributed to an Isospora ohioensis-like organism: A case report. Journal of the American Veterinary Medical Association. 173: 185-191.

Ferguson, D. J. P., A. Birch-Andersen, W. M. Hutchinson, And J. C. SiIm. 1980. Ultrastructural observations showing enteric multiplication of Cystoisospora (Isospora) felis by endodyogeny. Zeitshrift für Parasitenkunde 63: 289-291.

Hilali, M., F. A. Ghaffar, and E. Scholtyseck. 1979. Ultrastructural study of the endogenous stages of Isospora canis (Nemeséri, 1959) in the small intestine of dogs. Acta Veterinaria Academiae Scientiarum Hungaricae 27: 233-243. 
Lepp, D. L., AND K. S. TodD. 1974. Life cycle of Isospora canis Nemeséri, 1959 in the dog. Journal of Protozoology 21: 199206.

Levine, N. D. 1973. Protozoan parasites of domestic animals and of man, 2nd ed. Burgess Publishing Company, Minneapolis, Minnesota, $406 \mathrm{p}$.

Lindsay, D. S., A. E. Houk, S. M. Mitchell, and J. P. Dubey. 2014. Developmental biology of Cystoisospora (Apicomplexa: Sarcocystidae) monozoic tissue cysts. Journal of Parasitology 100: 392-398.
Lindsay, D. S., B. P. Stuart, B. E. Wheat, and J. V. Ernst. 1980. Endogenous development of the swine coccidium, Isospora suis Biester 1934. Journal of Parasitology 66: 771779.

Mitchell, S. M., A. M. Zajac, S. Charles, R. B. Duncan, and D. S. Lindsay. 2007. Cystoisospora canis Nemeséri, 1959 (syn. Isospora canis), infections in dogs: Clinical signs, pathogenesis, and reproducible clinical disease in beagle dogs fed oocysts. Journal of Parasitology 93: 345-352.

SнAн, H. L. 1971. The life cycle of Isospora felis Wenyon, 1923, a coccidium of the cat. Journal of Protozoology 18: 3-17. 